\title{
COUNTABLE DIRECT SUMS OF TORSION COMPLETE GROUPS
}

\author{
JOHN M. IRWIN, FRED RICHMAN AND ELBERT A. WALKER
}

1. Introduction. All groups considered in the sequel are abelian $p$-groups without elements of infinite height. Topological notions refer to the $p$-adic topology. Notation and terminology follow [2], the main exception being that groups in which every bounded Cauchy sequence converges are called torsion complete, the word "closed" being reserved for its topological sense.

In [4] Kolettis introduced the notion of a semicomplete group, i.e. a group which is the direct sum of a torsion complete group and a direct sum of cyclics, and showed that any two such decompositions had isomorphic refinements. These groups form a natural subject for investigation, subsuming the two classes of most understood groups. The more extensive class, playing the title role here, has the same attraction and is considerably less ad hoc. The main objective of this paper is to prove the isomorphic refinement theorem for any two decompositions of a group in this class.

2. Basic weapons. We begin with a lemma concerning extending purifications of subgroups of the socle of a torsion complete group.

Lemma 1. Let $G$ be a torsion complete group, $H$ a subgroup of $G[p]$, $K$ a pure subgroup of $G, K[p] \subseteq H$. There exists a pure subgroup $L$ of $G$ such that $K \subseteq L$ and $L[p]=H$.

Proof. By Zorn's lemma pick $L$ maximal among the pure subgroups of $G$ containing $K$ whose socles are contained in $H$. Suppose $x \in H-L$. Since $G$ is torsion complete, the reduced part of $G / L$ is torsion complete. Therefore $x+L$ is contained in a rank-one summand $S$ of $G / L$. The inverse image $S_{0}$ of $S$ is pure in $G$, contains $L$ and $x$, and $S_{0}[p] \subseteq H$, contradicting the maximality of $L$.

The next lemma reduces the notion of torsion completeness to the socle.

Lemma 2. Let $G$ be a p-group without elements of infinite height. Then $G$ is torsion complete if $G[p]$ is complete (in the G-topology).

Proof. We show by induction that $G\left[p^{n}\right]$ is complete. Let $\left\{x_{j}\right\}$ be a Cauchy sequence in $G\left[p^{n}\right]$. Then $\left\{p x_{j}\right\}$ is a Cauchy sequence in

Presented to the Society, April 24, 1965; received by the editors January 29, 1965. 
$G\left[p^{n-1}\right]$. By induction, $\left\{p x_{j}\right\}$ converges to some element $y=p z(p G$ is closed). Therefore $\left\{p\left(x_{j}-z\right)\right\}$ converges to 0 and we can find elements $g_{r} \in G$ and a subsequence $\left\{x_{j(r)}\right\}$ such that $p\left(x_{j(r)}-z\right)=p^{r} g_{r}$. Now $\left\{x_{j(r)}-z-p^{r-1} g_{r}\right\}$ is a Cauchy sequence in $G[p]$ and so converges to $h \in G[p]$. Therefore $\left\{x_{j(r)}\right\}$ and hence $\left\{x_{j}\right\}$ converges to $h+z$.

Combining these results we show our class to be closed under summands.

Theorem 1. Let $G=\sum_{1}^{\infty} G_{j}, G_{j}$ torsion complete. Suppose $H$ is a summand of $G$. Then $H=\sum_{1}^{\infty} H_{j}, H_{j}$ torsion complete.

Proof. By Lemma 1 we can define a chain of pure subgroups $\left\{T_{i}\right\}$ satisfying $T_{i} \subseteq \sum_{1}^{i} G_{j}$ and $T_{i}[p]=H[p] \cap \sum_{1}^{i} G_{j}$. But $T_{i}[p]$ is closed in $G$, being the intersection of two closed subgroups, and hence in $\sum_{1}^{i} G_{j}$. Since $\sum_{1}^{i} G_{j}$ is torsion complete and $T_{i}$ is pure, $T_{i}[p]$ is complete in $T_{i}$ and so, by Lemma $2, T_{i}$ is torsion complete. Let $\prod_{I I}$ be the projection of $G$ onto $H$. Then $\prod_{H} T_{i} \cong T_{i}$ is torsion complete and pure in $H$ (socle elements have correct heights) and therefore is a summand of $H$. We thus have a chain of torsion complete summands of $H, \prod_{H} T_{1} \subseteq \prod_{H} T_{2} \subseteq \cdots$ such that $U \prod_{H} T_{i}=H$ (since it is pure and contains $H[p])$. Define $H_{j}$ such that $\prod_{H} T_{j-1} \oplus H_{j}=\prod_{H} T_{j}$.

3. Isomorphic refinements. We hasten to the key lemma for the isomorphic refinement theorem, showing that a torsion complete summand of a direct sum of torsion complete groups can be pulled out piece by piece. First a technical matter.

Lemma 3. Let $G=\sum_{J} A_{j}, J$ an arbitrary index set. If $H$ is a torsion complete summand of $G$ then there exists a finite subset $I$ of $J$ such that $H[p]-\sum_{I} A_{j}$ has bounded height.

Proof. Suppose not. Select finite subsets $I_{1} \subset I_{2} \subset \cdots$ of $J$ and elements $x_{n} \in H[p]-\sum_{I_{n}} A_{j}$ such that $x_{n} \in H[p] \cap \sum_{I_{n+1}} A_{j}$ and $x_{n}$ has height exceeding $p^{n}$. By virtue of Lemma 1 there exists a chain $\left\{H_{n}\right\}$ of pure subgroups of $H$ satisfying $H_{n}[p]=H[p] \cap \sum_{I_{n}} A_{j}$. As in the proof of Theorem 1, each $H_{n}$ is torsion complete. By the same token $\mathrm{U} H_{n}$ is torsion complete. Thus $H_{n+1}=H_{n} \oplus H_{n}^{\prime}$ and $H_{n}^{\prime}$ has a nonzero element of height exceeding $p^{n}$, namely $\prod H_{n}^{\prime}\left(x_{n}\right)$. But $\bigcup H_{n}=H_{1} \oplus H_{1}^{\prime} \oplus H_{2}^{\prime} \oplus \cdots$, a contradiction.

Lemma 4. Let $G=\sum_{J} A_{j}, J$ an arbitrary index set, $A_{j}$ torsion complete. Let $H$ be a torsion complete summand of $G$. Then $A_{j}=A_{j}^{1} \oplus A_{j}^{2}$, $H \cong \sum_{J} A_{j}^{1}, G / H \cong \sum_{J} A_{j}^{2}$. 
Proof. We shall say that an arbitrary summand $H$ of $G$ can be pulled out of $\sum_{J} A_{j}$ if the $A_{j}$ 's decompose as above. The proof divides in to three parts.

(1) If $H \subseteq \sum_{I} A_{j}, I$ a finite subset of $J$, then $H$ can be pulled out. It is clearly enough to show that $H$ can be pulled out of $\sum_{I} A_{j}$. Since everything in sight is torsion complete, this follows from Corollary 34.5 of $[2]$.

(2) If $H$ is bounded, $H$ can be pulled out. This is immediate upon examining the appropriate maximal $p^{n}$-bounded summands and their complements (which are necessarily isomorphic, see [1]).

(3) Let $I$ be as in Lemma 3, $T$ a pure subgroup of $\sum_{I} A_{j}$ such that $T[p]=H[p] \cap \sum_{I} A_{j}($ Lemma 1$)$, and $K=\prod_{H} T$. We note, as in the proof of Theorem 1, that $T$ is torsion complete and isomorphic to $K$ which is pure in $H$. Now $H=K \oplus H_{0}$ where $H_{0}$ is bounded. Therefore $G / H \oplus K \oplus H_{0} \cong G$ and we may assume (replacing $K$ by $T$ using Theorem 17 of [3]) that $K \subseteq \sum_{I} A_{j}$. Thus we can pull $K$ and then $H_{0}$ out and hence can pull out $H$.

We are now in a position to prove the bulk of the refinement theorem.

THEOREM 2. Let $G$ be a direct sum of countably many torsion complete groups. Any two decompositions of $G$ into countably many groups have isomorphic refinements.

Proof. Suppose $G \cong \sum_{1}^{\infty} A_{i} \cong \sum_{1}^{\infty} B_{j}$. By Theorem 1 we can assume that $A_{i}$ and $B_{j}$ are torsion complete. We shall construct groups $A_{i j}$ and $B_{i j}$ such that $A_{i} \cong \sum_{j}\left(A_{i j} \oplus B_{i j}\right)$ and $B_{j} \cong \sum_{i}\left(A_{i j} \oplus B_{i j}\right)$. In the interest of clarity we shall not formalize the construction but give the first few steps and indicate the general procedure. By Lemma 4 we can pull $A_{1}$ out of $\sum_{1}^{\infty} B_{j} ; A_{1}=\sum_{1}^{\infty} A_{1 j}, B_{j} \cong A_{1 j} \oplus B_{j}^{\prime}$, leaving $\sum_{2}^{\infty} A_{i} \cong \sum_{1}^{\infty} B_{j}^{\prime}$. We now pull $B_{1}^{\prime}$ out of $\sum_{2}^{\infty} A_{i} ; B_{1}^{\prime}$ $=\sum_{2}^{\infty} B_{i 1}, A_{i} \cong B_{i 1} \oplus A_{i}^{\prime}$, leaving $\sum_{2}^{\infty} A_{i}^{\prime} \cong \sum_{2}^{\infty} B_{j}^{\prime}$. Now pull $A_{2}^{\prime}$ out of $\sum_{2}^{\infty} B_{j}^{\prime}$ etc., crisscrossing back and forth and exhausting both decompositions.

We note the following corollary concerning semicomplete groups which follows immediately.

Corollary. If $G$ is a semicomplete group so is any summand of $G$.

We now have the refinement theorem for countable decompositions. The next lemma, which parallels well known facts about decomposing torsion complete groups, says that all decompositions are essentially countable. 
Lemma 5. Let $G$ be a direct sum of countably many torsion complete groups. If $G=\sum B_{j}$ then all but a countable number of the $B_{j}$ 's are bounded.

Proof. By Theorem 1 we may assume the $B_{j}$ 's are torsion complete, that there are uncountably many of them and that none are bounded. Let $G=\sum_{1}^{\infty} A_{i}, A_{i}$ torsion complete. Since $B_{j}$ is unbounded, there exists a positive integer $n$ such that $B_{j}\lfloor p] \cap \sum_{1}^{n} A_{i}$ has unbounded height. Since there are uncountably many $B_{j}$ 's, there is some $n$ for which this holds for infinitely many $B_{j}$ 's. This contradicts Lemma 3.

THEOREM 3. Let $G$ be a direct sum of countably many torsion complete groups. Any two decompositions of $G$ have isomorphic refinements.

Proof. Let $G=\sum_{I} A_{s}=\sum_{J} B_{i}$. Theorem 1 and Lemma 5 allow us to assume that the $A_{s}$ 's and $B_{t}$ 's are torsion complete and all but a countable number of them are bounded. Relabeling, $\sum_{I} A_{8}$ $=\sum_{1}^{\infty} A_{i} \oplus \sum_{K} C_{s}=\sum_{J} B_{t}=\sum_{1}^{\infty} B_{j} \oplus \sum_{L} D_{t}$, where each $A_{i}$ and $C_{s}$ is an $A_{s}$, the $C_{s}$ 's are bounded, and similarly for the $B_{j}$ 's and $D_{t}$ 's. We may further assume that each $C_{s}$ and $D_{t}$ is cyclic. Let $C=\sum C_{s}$, $D=\sum D_{i}$. By Theorem 2, the decompositions $\sum_{1}^{\infty} A_{i} \oplus C=\sum_{1}^{\infty} B_{j} \oplus D$ have isomorphic refinements $\sum E_{u}=\sum F_{u}$. We may assume that if $E_{u}$ is a summand of $C$ then $E_{u}=C_{\varepsilon}$ for some $s$, and similarly for $F_{u}$. Thus each $E_{u}$ is a summand of an $A_{s}, s \in I$, and each $F_{u}$ is a summand of a $B_{t}, t \in J$, completing the proof.

\section{REFERENCES}

1. D. O. Cutler, Quasi-isomorphisms for infinite abelian groups, Pacific I. Math. (to appear).

2. L. Fuchs, Abelian groups, Pergamon Press, Oxford, 1960.

3. J. M. Irwin and E. A. Walker, On N-high subgroups of abelian groups, Pacific J. Math. 11 (1961), 1363-1374.

4. G. Kolettis, Semi-complete primary abelian groups, Proc. Amer. Math. Soc. 11 (1960), 200-205.

New Mexico State University 